\title{
Classification of mobile- and immobile-molecule timescales for the Stokes-Einstein and Stokes-Einstein-Debye relations in supercooled water
}

\author{
Takeshi Kawasaki ${ }^{1}$ and Kang Kim ${ }^{2}$ \\ ${ }^{1}$ Department of Physics, Nagoya University, Nagoya 464-8602, Japan \\ ${ }^{2}$ Division of Chemical Engineering, Graduate School of Engineering Science, Osaka \\ University, Toyonaka, Osaka 560-8531, Japan \\ E-mail: kawasaki@r.phys.nagoya-u.ac.jp and kk@cheng.es.osaka-u.ac.jp
}

\begin{abstract}
Molecular dynamics simulations have been performed on TIP4P/2005 supercooled water to investigate the molecular diffusion and shear viscosity at various timescales and assess the Stokes-Einstein (SE) and Stokes-Einstein-Debye (SED) relations. For this purpose, we calculated various time correlation functions, such as the mean-squared displacement, stress relaxation function, density correlation function, hydrogen-bond correlation function, rotational correlation function of molecular orientation, non-Gaussian parameter, and four-point correlation function. Our study of the SE and SED relations indicates that the transport coefficients and timescales obtained using these time correlation functions may be classified into two distinct classes: those governed by either mobile or immobile molecules, due to dynamical heterogeneity. In particular, we show that the stress relaxation time, hydrogen-bond lifetime, and large-angle rotational relaxation time are coupled with translational diffusion, and are characterized by mobile molecules. In contrast, the structural $\alpha$ relaxation time, small-angle rotational relaxation time, and characteristic timescales of four-point correlation functions are decoupled with translational diffusion, and are governed by immobile molecules. This decoupling results in a violation of the $\mathrm{SE}$ relation. These results indicate that the identification of timescales that appropriately characterize transport coefficients, such as translational diffusion constant and shear viscosity, provides a deep insight into the violation of the SE and SED relations in glass-forming liquids.
\end{abstract}

Keywords: slow relaxation and glassy dynamics, Molecular dynamics, Dynamical heterogeneities

\section{Introduction}

The need for a unified description of the structural relaxation mechanism in various glass-forming liquids is one of the fundamental problems of condensed matter physics [1, 
2]. Scattering and spectroscopic experiments provide data for various timescales, such as the structural $\alpha$-relaxation time $\tau_{\alpha}$ of the density correlation function and relaxation times for molecular reorientation $\tau_{\ell}$, obtained using the $\ell$-th order of the Legendre polynomial. Moreover, transport coefficients such as shear viscosity and diffusion constant play a crucial role in characterizing the slow dynamics of glass-forming liquids in the vicinity of the glass transition temperature [3, 4]

The Stokes-Einstein (SE) relation, $D_{\mathrm{t}}^{-1} \propto \eta / T$, and the Stokes-Einstein-Debye (SED) relation, $\tau_{\ell} \propto \eta / T$ (or $D_{\mathrm{t}}^{-1} \propto \tau_{\ell}$ ), are thought to comprise the key characteristics necessary to provide the required unified description of structural relaxation. Here, $T$, $\eta$, and $D_{\mathrm{t}}$ denote the temperature, the shear viscosity, and the translational diffusion constant, respectively. In fact, these SE and SED relations typically break down in the case of supercooled states, which is regarded as a hallmark of the spatially heterogeneous dynamics that are characterized by the non-Gaussian and the non-exponential nature of various time correlation functions [2, 5, 6]. In particular, the violations of SE and SED relations indicate decouplings between the molecular diffusion and the shear viscosity 7 10]. Thus, it is necessary to reveal the link between transport coefficients and the characteristic timescales, and many theoretical studies have been devoted to this issue [11, 12].

Molecular dynamics (MD) simulations of model glass-forming liquids have been previously conducted in order to investigate the violation of the SE relation [13 31]. Whether the temperature dependence of shear viscosity $\eta$ may be replaced by the $\alpha$ relaxation time $\tau_{\alpha}$ is a controversial issue [22]. However, numerical calculations of $\eta$ have demonstrated $\tau_{\alpha} \propto \eta / T$ in both fragile and strong glass-formers, indicating that $D_{\mathrm{t}} \tau_{\alpha}$ is a good indicator for $D_{\mathrm{t}} \eta / T$, even in supercooled states [15, 25]. The violation of the SED relation in supercooled molecular liquids has also been examined using MD simulations [32 34]. However, there has been no direct assessment of the quantity $\tau_{\ell} T / \eta$ through the variation of $\ell$. Instead, the temperature dependence of $D_{\mathrm{t}} \tau_{\ell}$ with $\ell=2$ has been mainly quantified, which is comparable to the results of experimental analysis [5, 6]. Furthermore, the connection between $\tau_{\alpha}$ and $\tau_{\ell}$ for varying the degree $\ell$ remains elusive, particularly in supercooled states, and thus particular care should be taken when discussing the coupling of translational and rotational molecular motions.

The system of supercooled water has attracted much attention owing to its capacity for elucidating the mechanism underlying SE and SED relations through both experiments and simulations 35 55. Comprehensive numerical calculations of the shear viscosity enabled the precise evaluation of SE and SED relations [51, 55]. In particular, it was demonstrated that the shear viscosity $\eta$ can be represented by the approximation $\eta \approx G_{\mathrm{p}} \tau_{\eta} \Gamma\left(1 / \beta_{\eta}\right) / \beta_{\eta}$, with the Gamma function $\Gamma(x)$, according to the approximation, $G_{\eta}(t) \approx G_{\mathrm{p}} \exp \left\{-\left(t / \tau_{\eta}\right)^{\beta_{\eta}}\right\}$, for the long time behavior of $G_{\eta}(t)$ [51]. Here, $G_{\mathrm{p}}, \tau_{\eta}$, and $\beta_{\eta}$ denote the plateau modulus, the stress relaxation time, and the degree of non-exponentiality of the stress correlation function $G_{\eta}(t)$, respectively. In contrast, the translational diffusion constant $D_{\mathrm{t}}$ is governed by hydrogen-bond (Hbond) breakage processes, leading to the proportional relationship of $D_{\mathrm{t}}^{-1} \propto \tau_{\mathrm{HB}}$ 
with the H-bond lifetime, $\tau_{\mathrm{HB}}$ [51]. An analogous relationship has been demonstrated in both fragile supercooled liquids [24] and silica-like strong supercooled liquids [25], where the bond-breakage method was used to characterize changes in local connectivity of molecules [14, 56 59]. Furthermore, we previously conducted a comprehensive investigation into the SED relations, $\tau_{\ell} T / \eta$ and $D_{\mathrm{t}} \tau_{\ell}$, for various $\tau_{\ell}$ [55]. It was demonstrated that these SED relations shows a strong dependence on the degree $\ell$ : the higher-order $\tau_{\ell}$ values exhibit a temperature dependence similar to that of $\eta / T$, whereas the lowest-order $\tau_{\ell}$ values are coupled with $D_{\mathrm{t}}$.

In this study, we examine the roles of characteristic timescales, including the $\alpha$ relaxation time $\tau_{\alpha}, \mathrm{H}$-bond lifetime $\tau_{\mathrm{HB}}$, rotational relaxation times $\tau_{\ell}(\ell=1$ and 6$)$, stress relaxation time $\tau_{\eta}$, and the timescales of non-Gaussian parameters and four-point dynamic correlations, in the SE and SED relations. In particular, the aim is to classify these timescales into two classes: those are coupled or decoupled with $D_{\mathrm{t}}$ (or $\eta / T$ ). We also discuss this classification in terms of the mobile/immobile contributions of the dynamic heterogeneities in supercooled water. The rest structure of the paper is organized as follows: In Sec. 2, we describe the MD simulations of supercooled water using the TIP4P/2005 model. In Sec. 3, we describe the numerical calculations of various timescales and discuss their temperature dependence, compared with those of $D_{\mathrm{t}}$ and $\eta / T$. In Sec. 4, we summarize our conclusions.

\section{MD Simulations}

We performed MD simulations of liquid water using the Large-scale Atomic/Molecular Massively Parallel Simulator (LAMMPS) [60], and used the TIP4P/2005 water model [61]. There are various studies in the literature of MD simulations used to investigate various properties in supercooled states of this model [53, 54, 62, 69]. In the present study, in order to obtain equilibrated initial configurations, we performed simulations with the $N V T$ ensembles for $N=1,000$ water molecules at various temperatures $(T=300,280,260,250,240,230,220,210,200$, and $190 \mathrm{~K})$ at a fixed mass density of $\rho=1 \mathrm{~g} \mathrm{~cm}^{-3}$. The corresponding linear dimension was $L=31.04$ $\AA$. The $N V E$ ensemble simulations were conducted after the equilibration, remaining at each temperature for a sufficiently long-time periods, from which the various time correlation functions were calculated. Periodic boundary conditions were utilized in all the simulations, with a simulation time step of 1 fs. 

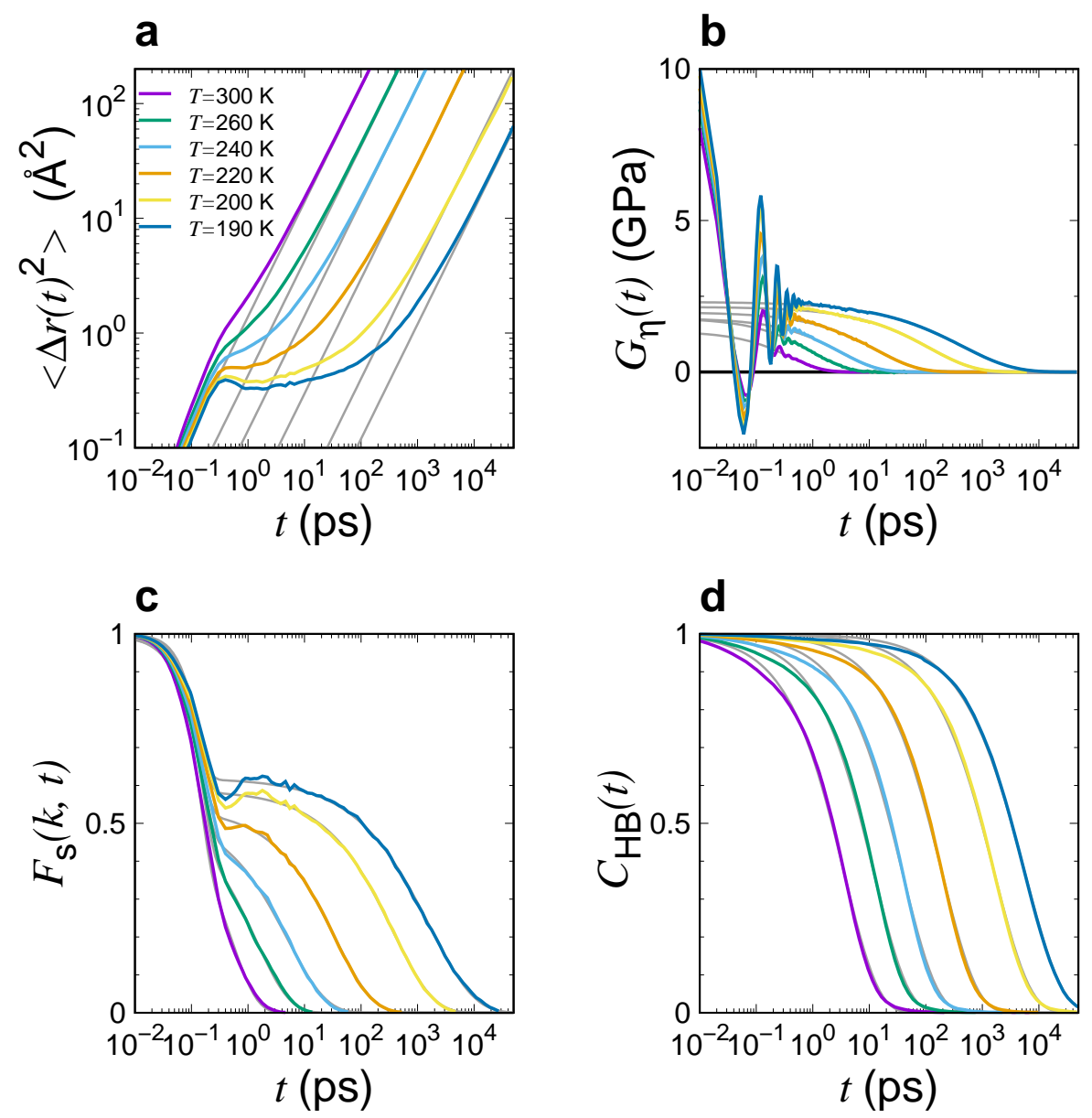

Figure 1. (a) Mean-squared displacement $\left\langle\Delta r(t)^{2}\right\rangle$. Gray solid lines represent $6 D_{\mathrm{t}} t$ obtained by the fitting of the long time region in $\left\langle\Delta r(t)^{2}\right\rangle$. (b) The Stress correlation function $G_{\eta}(t)$. Stress relaxation time $\tau_{\eta}$ is determined by the fitting $G_{\eta}(t)$ with the KWW function, $G_{\mathrm{p}} \exp \left\{-\left(t / \tau_{\eta}\right)^{\beta_{\eta}}\right\}$. Gray solid lines represent the fitting results. (c) The incoherent intermediate scattering function $F_{\mathrm{s}}(k, t)$. The structural $\alpha$-relaxation time $\tau_{\alpha}$ is determined by the fitting $F_{\mathrm{S}}(k, t)$ with the KWW function $\left(1-f_{\mathrm{c}}\right) \exp \left[-\left(t / \tau_{\mathrm{s}}\right)^{2}\right]+f_{\mathrm{c}} \exp \left[-\left(t / \tau_{\alpha}\right)^{\beta_{\alpha}}\right]$. Gray solid lines represent the fitting results. (d) H-bond correlation function $C_{\mathrm{HB}}(t)$. The H-bond lifetime $\tau_{\mathrm{HB}}$ is determined by fitting $C_{\mathrm{HB}}(t)$ with $\exp \left[-\left(t / \tau_{\mathrm{HB}}\right)^{\beta_{\mathrm{HB}}}\right]$. Gray solid lines represent the fitting results.

\section{Results and discussion}

\subsection{Mean-squared displacement and translational diffusion constant}

As an important component of the SE relation, we quantified the translational diffusion constant $D_{\mathrm{t}}$. To this end, we calculated the mean squared displacement,

$$
\left\langle\Delta r(t)^{2}\right\rangle=\left\langle\frac{1}{N} \sum_{j=1}^{N}\left|\mathbf{r}_{j}(t)-\mathbf{r}_{j}(0)\right|^{2}\right\rangle,
$$

where $\mathbf{r}_{j}(t)$ represents the position of $j$-th $\mathrm{O}$ atom at time $t$. The bracket $\langle\cdots\rangle$ indicates an average over the initial time 0 . In Fig. 1(a), $\left\langle\Delta r(t)^{2}\right\rangle$ is plotted for 

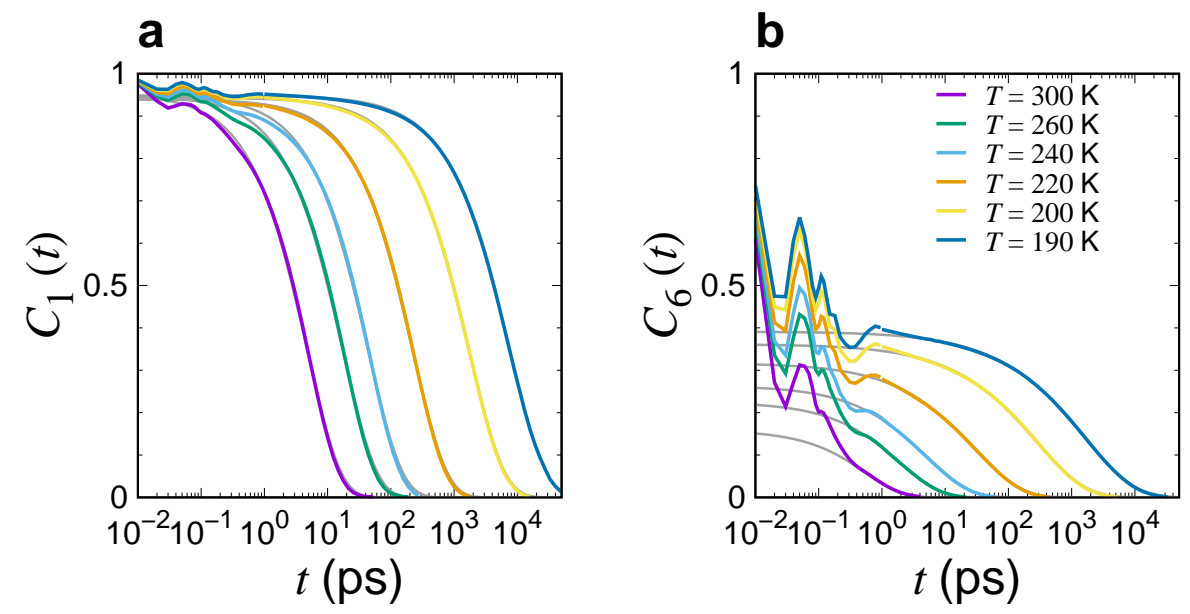

Figure 2. Rotational correlation functions $C_{\ell}(t)$ with $\ell=1$ (a) and $\ell=6$ (b). The rotational relaxation time $\tau_{\ell}$ is determined by fitting $C_{\ell}(t)$ with the KWW function $A_{\ell} \exp \left\{-\left(t / \tau_{\ell}\right)^{\beta_{\ell}}\right\}$.
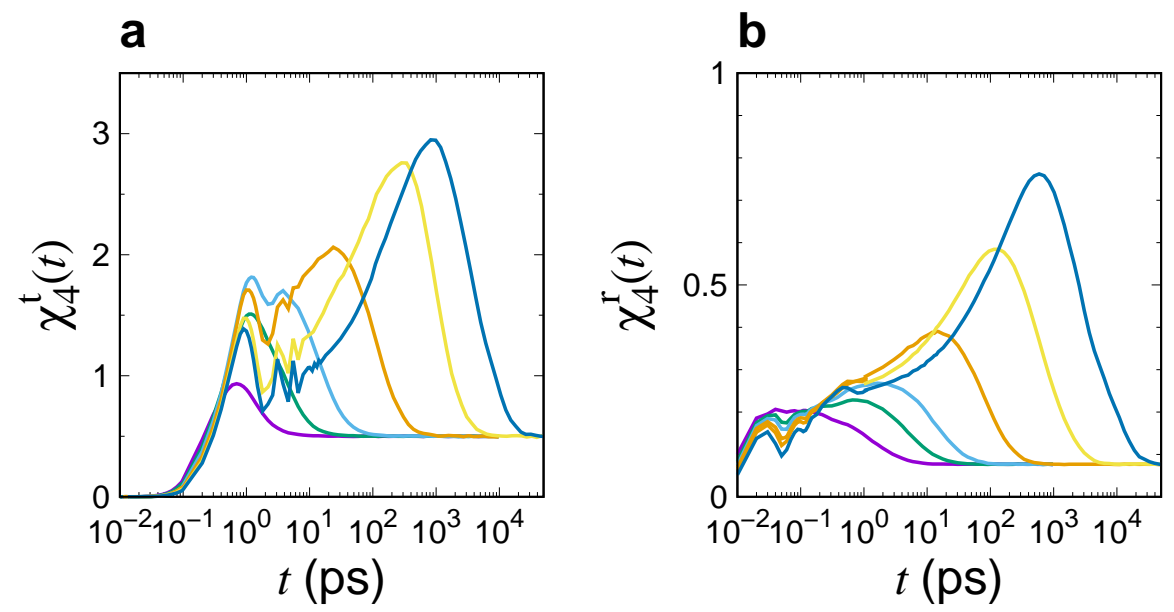

Figure 3. Four-point correlation functions of translational (a) and rotational (b) motion. The peak times of $\chi_{4}^{\mathrm{t}}(t)$ and $\chi_{4}^{\mathrm{r}}(t)$ are denoted by $\tau_{\mathrm{t}}$ and $\chi_{4}^{\mathrm{r}}(t)$, respectively.

various temperatures. For each temperature, the ballistic behavior, $\left\langle\Delta r(t)^{2}\right\rangle \propto t^{2}$, is observed in the short time region. The diffusive behavior, $\left\langle\Delta r(t)^{2}\right\rangle \propto t$, eventually develops in the long time region. The plateau that is observed in the intermediate region is pronounced upon supercooling, indicating cage effects related to the presence of neighboring molecules. The translational diffusion constant $D_{\mathrm{t}}$ is determined from the long time behavior of $\left\langle\Delta r(t)^{2}\right\rangle$ using the Einstein relation, $D_{\mathrm{t}}=\lim _{t \rightarrow \infty}\left\langle\Delta r(t)^{2}\right\rangle / 6 t$. The solid lines in Fig. (1) (a) are the results of the fitting with $\left\langle\Delta r(t)^{2}\right\rangle=6 D_{\mathrm{t}} t$. 

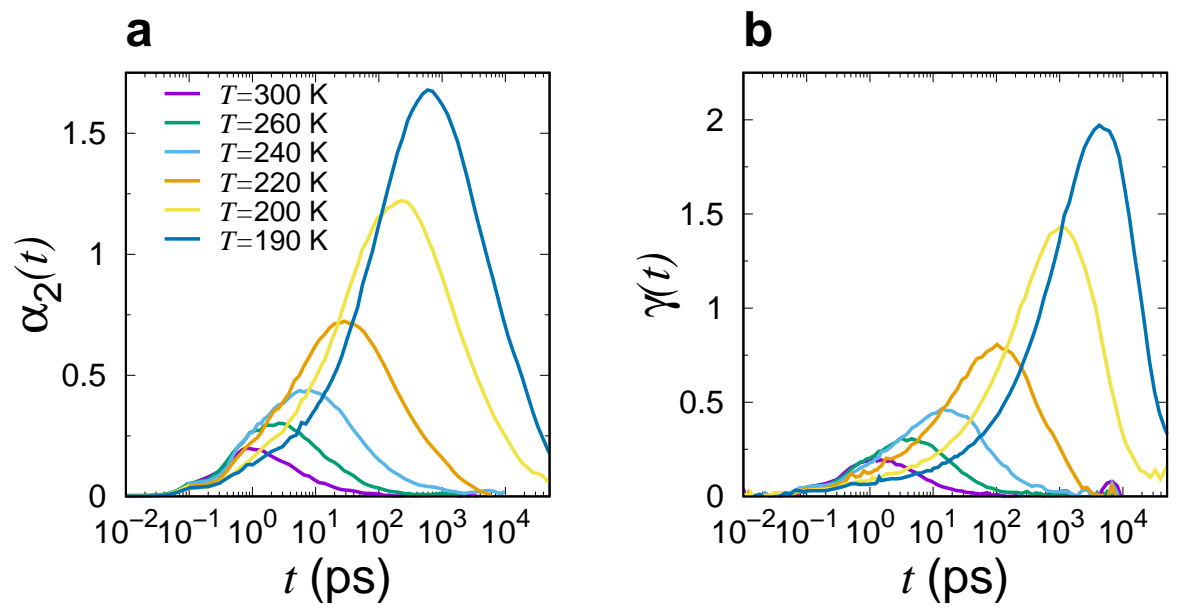

Figure 4. Non-Gaussian parameter $\alpha_{2}(t)$ (a) and new non-Gaussian parameter $\gamma(t)$ (b). The peak times of $\alpha_{2}(t)$ and $\gamma(t)$ are denoted by $\tau_{\text {ngp }}$ and $\tau_{\text {nngp }}$, respectively.

a

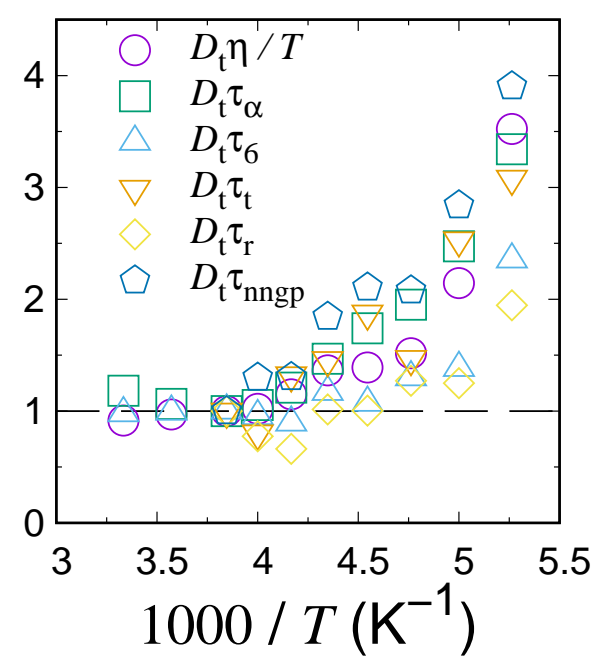

b

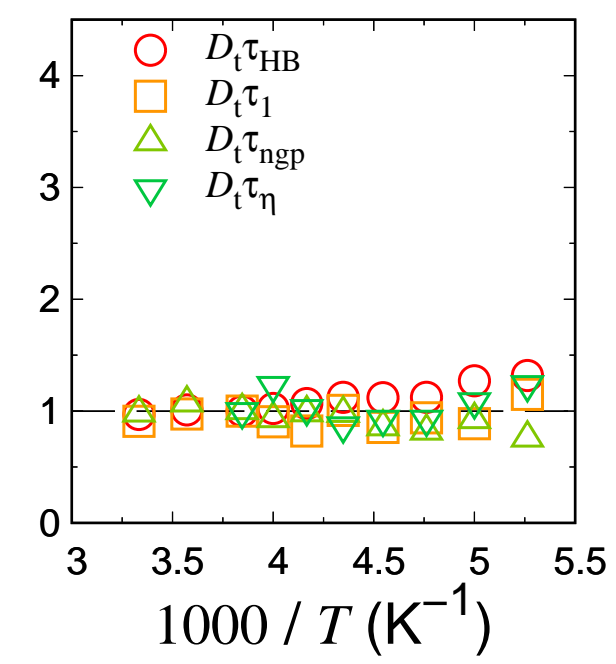

Figure 5. (a) Inverse temperature 1000/T dependence of the scaled SE ratios, obtained using shear viscosity divided by temperature $\eta / T$, structural $\alpha$-relaxation time $\tau_{\alpha}$, rotational relaxation time $\tau_{6}$, peak time of the translational four-point correlation function $\tau_{\mathrm{t}}$, peak time of the rotational four-point correlation function $\tau_{\mathrm{r}}$, and peak time of NNGP $\tau_{\text {nngp }}$. All quantities are scaled by their values at 260 K. (b) Inverse temperature 1000/T dependence of the scaled SE ratios obtained using H-bond life time $\tau_{\mathrm{HB}}$, rotational relaxation time $\tau_{1}$, peak time of NGP $\tau_{\text {ngp }}$, and stress relaxation time $\tau_{\eta}$. All quantities are scaled by their values at $260 \mathrm{~K}$.

\subsection{Stress correlation function and shear viscosity}

In order to obtain the shear viscosity $\eta$ as another essential component of the SE relation, the autocorrelation function of the off-diagonal stress tensor was calculated, which is 
given by

$$
G_{\alpha \beta}(t)=\frac{V}{k_{\mathrm{B}} T}\left\langle\sigma_{\alpha \beta}(t) \sigma_{\alpha \beta}(0)\right\rangle,
$$

where $V$ is the volume of the system and $\sigma_{\alpha \beta}$ represents the $\alpha \beta(=x, y, z)$ components of the off-diagonal stress tensor. The average stress correlation function is then defined as $G_{\eta}(t)=\left(G_{x y}(t)+G_{x z}(t)+G_{y z}(t)\right) / 3$. $G_{\eta}(t)$ is plotted in Fig. 1 (b), for various temperatures. The instantaneous time correlation $G_{\eta}(0)$ corresponds to the instantaneous or affine shear modulus $G_{\infty}$. In the short time region, $G_{\eta}(t)$ shows large fluctuations, which are attributed to vibrational motions observed in network forming liquids such as silica glass [25]. The $G_{\eta}(t)$ plateaus in the intermediate time region, particularly at lower temperatures, which is the so-called the plateau modulus. $G_{\eta}(t)$ finally decays to zero at longer timescales. The shear viscosity $\eta$ is determined from the integral of $G_{\eta}(t)$ as

$$
\eta=\int_{0}^{\infty} G_{\eta}(t) \mathrm{d} t
$$

using the Green-Kubo formula. Furthermore, we examine the stress relaxation time $\tau_{\eta}$ of $G_{\eta}(t)$. The long time behavior of $G_{\eta}(t)$ is fitted using the Kohlrausch-WilliamsWatts (KWW) stretched-exponential function, $G_{\mathrm{p}} \exp \left\{-\left(t / \tau_{\eta}\right)^{\beta_{\eta}}\right\}$. Here, $G_{\mathrm{p}}$ and $\tau_{\eta}$ denote the plateau modulus and the stress relaxation time, respectively. The exponent $\beta_{\eta}(<1)$ relates to the degree of non-exponentiality of $G_{\eta}(t)$. The gray solid lines in Fig. 1(b) indicate those fitting results.

The SE ratio $D_{\mathrm{t}} \eta / T$ as a function of the inverse of the temperature is displayed in Fig. 5(a). This plot demonstrates the violation of the SE relation for temperatures below $240 \mathrm{~K}$.

\subsection{Incoherent intermediate scattering function and $\alpha$-relaxation time}

As an alternative to $\eta$, the structural $\alpha$-relaxation time $\tau_{\alpha}$ has been frequently used in discussions of the violation of the SE relation, which is obtained from the incoherent intermediate scattering function given by

$$
F_{\mathrm{S}}(k, t)=\left\langle\frac{1}{N} \sum_{j=1}^{N} \exp \left[i \mathbf{k} \cdot\left(\mathbf{r}_{j}(t)-\mathbf{r}_{j}(0)\right)\right]\right\rangle \text {. }
$$

Here, $\mathbf{r}_{j}(t)$ is the position vector of the $\mathrm{O}$ atom of the water molecule $j$ at time $t$. The wave number $k=|\mathbf{k}|$ was set to $k=3.0 \AA^{-1}$, which corresponds to the first peak position of the static structure factors $S(k)$ of the $\mathrm{O}$ atom. $F_{\mathrm{s}}(k, t)$ is plotted for various temperatures in Fig. $1(\mathrm{c})$. A two-step relaxation in $F_{s}(k, t)$ is observed upon supercooling. The $\alpha$-relaxation time $\tau_{\alpha}$ is determined by the fitting $F_{\mathrm{s}}(k, t)$ with the KWW function, i.e., $\left(1-f_{\mathrm{c}}\right) \exp \left[-\left(t / \tau_{\mathrm{s}}\right)^{2}\right]+f_{\mathrm{c}} \exp \left[-\left(t / \tau_{\alpha}\right)^{\beta_{\alpha}}\right]$, where $f_{\mathrm{c}}, \tau_{\mathrm{s}}, \tau_{\alpha}$, and $\beta_{\alpha}$ are the fitting parameters [23]. The exponent $\beta_{\alpha}$ is the degree of non-exponentiality of $F_{\mathrm{s}}(k, t)$. 
The quantity $D_{\mathrm{t}} \tau_{\alpha}$ is plotted in Fig. 5(a) for various temperatures. These results show that the violation of the SE relation is explained in terms of $D_{\mathrm{t}} \tau_{\alpha}$, suggesting the proportional relationship, $\tau_{\alpha} \propto \eta / T$.

\subsection{Hydrogen-bond breakage and its lifetime}

Next, we focus on the H-bond life time $\tau_{\mathrm{HB}}$, which is thought to be a type of structural relaxation of molecules in the case of liquid water. However, it will be demonstrated that $\tau_{\mathrm{HB}}$ behaves differently from $\eta / T$ and $\tau_{\alpha}$. The dynamics of H-bond network rearrangement was investigated using the correlation function,

$$
C_{\mathrm{HB}}(t)=\left\langle\sum_{i, j}^{N} h_{i j}(0) h_{i j}(t)\right\rangle /\left\langle\sum_{i, j}^{N} h_{i j}(0) h_{i j}(0)\right\rangle \text {, }
$$

where $h_{i j}(t)$ denotes the H-bond indicator between $i$-th and $j$-th molecules at a time $t$ 70 74. In this study, the H-bond is defined by the r-definition, where only the intermolecular $\mathrm{O}-\mathrm{H}$ distance $r_{\mathrm{OH}}$ is investigated [75]. $h_{i j}(t)=1$ if the $r_{\mathrm{OH}}$ between the $i$-th and $j$-th molecules is less than $2.4 \AA$, corresponding to the first minimum of the radial distribution function $g_{\mathrm{OH}}(r)$, and $h_{i j}(t)=0$ otherwise. The time dependence of $C_{\mathrm{HB}}(t)$ is given in Fig. 1(d), where the decay time of $C_{\mathrm{HB}}(t)$ increases with decreasing the temperature. However, they do not show the two-step relaxation for all temperatures, contrary to the observations of $F_{\mathrm{s}}(k, t)$. The H-bond lifetime $\tau_{\mathrm{HB}}$ is determined by the

fitting $C_{\mathrm{HB}}(t)$ with the KWW functions, $\exp \left\{-\left(t / \tau_{\mathrm{HB}}\right)^{\beta_{\mathrm{HB}}}\right\}$, where the exponent $\beta_{\mathrm{HB}}$ is the degree of non-exponentiality of $C_{\mathrm{HB}}(t)$. The gray solid lines in Fig. 1](d) indicate the results of the KWW fittings. Note that the H-bond correlation function $C_{\mathrm{HB}}$ has a form that is essentially identical to the bond-breakage method, which has been applied to various supercooled liquids [14,24, 25, 56, 59]. These previous studies have demonstrated that the bond-breakage method is a remarkable method for characterizing "genuine" configurational rearrangements in supercooled states.

Figure 5(b) provides the temperature dependence of $D_{\mathrm{t}} \tau_{\mathrm{HB}}$ for various temperatures. Remarkably, these plots reveal that $\tau_{\mathrm{HB}}$ is coupled with $D_{\mathrm{t}}$, which differs from $\tau_{\alpha}$. This result can be thought of as the preservation of the SE relation [51]. Furthermore, this preservation of the SE relation is interpreted in terms of the observation that $D_{\mathrm{t}}$ and $\tau_{\mathrm{HB}}$ are governed by molecules with jump/fast motions [24, 25, 51]. In contrast, we will show that $\eta$ and $\tau_{\alpha}$ are governed by immobile molecules with dynamic heterogeneities.

\subsection{Rotational relaxation times}

Molecular reorientations are often characterized by the rotational correlation function,

$$
C_{\ell}(t)=\frac{1}{N} \sum_{j=1}^{N}\left\langle P_{\ell}\left[\mathbf{e}_{j}(t) \cdot \mathbf{e}_{j}(0)\right]\right\rangle
$$


where $\mathbf{e}_{j}(t)$ is the normalized polarization vector of molecule $j$ and $P_{\ell}[x]$ is the $\ell$-th order Legendre polynomial as a function of $x$. In the present study, we examine the $\ell$-th $(\ell=1$ and 6$)$ order rotational relaxation function $C_{\ell}(t)$. Plots of $C_{\ell}(t)$ are provided in Fig. 2(a) and (b), with $\ell=1$ and 6, respectively. The gray solid lines represent the fitting results of the KWW function, $A_{\ell} \exp \left\{-\left(t / \tau_{\ell}\right)^{\beta_{\ell}}\right\}$. The rotational relaxation time $\tau_{1}$ and $\tau_{6}$ were quantified as a result of this fitting procedure.

The SED ratio $D_{\mathrm{t}} \tau_{6}$ is given in Fig. 5(a) as a function of the inverse of the temperature. This result shows the violation of the SE relation. However, another ratio $D_{\mathrm{t}} \tau_{1}$ indicates the preservation of the SED relation, as shown in Fig. 5(b). This conflicting result can be understood as follows: $\tau_{1}$ is characterized by the molecular reorientation related to large rotational movements, which are mostly large translational jump motions. Therefore, $\tau_{1}$ is coupled with $D_{\mathrm{t}}$. On the contrary, the small angular rotational movements characterizing $\tau_{6}$ are governed by "immobile" molecules, which is decoupled with $D_{\mathrm{t}}$, particularly at lower temperatures.

\subsection{Characterizations of dynamic heterogeneities - four-point correlation functions}

In order to elucidate the degree of dynamic heterogeneities and the related characteristic timescales in supercooled water, we examine the four-points correlation functions for translational and rotational motions [76]. The four-point correlation function $\chi_{4}^{\mathrm{t}}(t)$ for translational motion is defined by the variance of the intermediate scattering function $F_{s}(k, t)$ as,

$$
\chi_{4}^{\mathrm{t}}(t)=N\left[\left\langle\hat{F}_{s}(k, t)^{2}\right\rangle-\left\langle\hat{F}_{s}(k, t)\right\rangle^{2}\right],
$$

where $\hat{F}_{s}(t)=(1 / N) \sum_{j=1}^{N} \cos \left[\mathbf{k} \cdot \Delta \mathbf{r}_{j}(t)\right]$. The wave number $k=|\mathbf{k}|$ was set to $k=3.0$ $\AA^{-1}$. The rotational four-point correlation function $\chi_{4}^{\mathrm{r}}(t)$ can be analogously defined as

$$
\chi_{4}^{\mathrm{r}}(t)=N\left[\left\langle\hat{C}_{\ell}(t)^{2}\right\rangle-\left\langle\hat{C}_{\ell}(t)\right\rangle^{2}\right]
$$

where $\hat{C}_{\ell}(t)=(1 / N) \sum_{j=1}^{N} P_{\ell}\left[\mathbf{e}_{j}(t) \cdot \mathbf{e}_{j}(0)\right]$. The order of the Legendre polynomial was set to $\ell=6$. Figure 3 shows the temperature dependence of the four-point correlation functions, $\chi_{4}^{\mathrm{t}}(t)$ and $\chi_{4}^{\mathrm{r}}(t)$. The peak times of $\chi_{4}^{\mathrm{t}}(t)$ and $\chi_{4}^{\mathrm{r}}(t)$ are quantified and denoted by $\tau_{\mathrm{t}}$ and $\tau_{\mathrm{r}}$, respectively. We find that upon supercooling the peak heights increase, together with an increase in their peak times $\tau_{\mathrm{t}}$ and $\tau_{\mathrm{r}}$ are also increased.

Figure 5(a) shows a plot of $D_{\mathrm{t}} \tau_{t}$ and $D_{\mathrm{t}} \tau_{\mathrm{r}}$ as a function of the inverse of the temperature. Both quantities exhibit a temperature dependence similar to that observed in the case of the violation of the SE relation. This indicates that the characteristic timescales of translational and rotational dynamic heterogeneities are coupled with $\eta / T$ and $\tau_{\alpha}$.

\subsection{Characterizations of dynamic heterogeneities - non Gaussian parameters}

Finally, we elucidate the role of "mobile" and "immobile" molecules with dynamical heterogeneities on the SE and SED relations. First, we investigate the non-Gaussian 
parameter (NGP) that mainly characterizes the displacements of "mobile" molecules [77, 78. The equation is given by

$$
\alpha_{2}(t)=\frac{3}{5} \frac{\left\langle\Delta r(t)^{4}\right\rangle}{\left\langle\Delta r(t)^{2}\right\rangle^{2}}-1
$$

Second, to emphasize the effect of "immobile" molecules, another type of non-Gaussian parameter is defined by

$$
\gamma(t)=\frac{1}{3}\left\langle\Delta r(t)^{2}\right\rangle\left\langle\frac{1}{\Delta r(t)^{2}}\right\rangle-1
$$

which is referred to as the new non-Gaussian parameter (NNGP) [79]. Figure 4 shows the time evolutions of NGP $\alpha_{2}(t)$ and NNGP $\gamma(t)$ at various temperatures. The peak heights of $\alpha_{2}(t)$ and $\gamma(t)$ increase with decreasing the temperature. This means that the dynamics are more heterogeneous upon supercooling. Their peak times are denoted by $\tau_{\text {ngp }}$ and $\tau_{\text {nngp }}$, respectively. The corresponding temperature dependences $D_{\mathrm{t}} \tau_{\text {ngp }}$ and $D_{\mathrm{t}} \tau_{\text {nngp }}$ are given in Fig. 5(a) and Fig. 5(b), respectively. As shown in Fig. 5(a), the timescale of the "immobile" molecules, $\tau_{\text {nngp }}$, follows the temperature dependence observed for the violation of the SE relation, $D_{\mathrm{t}} \eta / T$ and $D_{\mathrm{t}} \tau_{\alpha}$. It is eventually concluded that the timescales, $\tau_{\alpha}, \tau_{6}, \tau_{\mathrm{t}}$, and $\tau_{\mathrm{r}}$ are dominated by "immobile" molecules of dynamic heterogeneities. By contrast, Fig. 5(b) demonstrates that $\tau_{\text {ngp }}$ follows the preservation of the $\mathrm{SE}$ relation. In this case, the timescales that are coupled with $D_{\mathrm{t}}, \tau_{\eta}, \tau_{\mathrm{HB}}$, and $\tau_{1}$, are dominated by "mobile" molecules with dynamic heterogeneities.

\section{Conclusion}

In this study, we performed MD simulations on supercooled liquid water using the TIP4P/2005 model. We determined the temperature dependence of transport coefficients such as the translational diffusion constant $D_{\mathrm{t}}$ and shear viscosity $\eta$, together with the timescales characterizing the dramatic slowing down of glassy dynamics. The SE relation, $D_{\mathrm{t}} \eta / T$, was also thoroughly assessed. The temperature dependence of two transport coefficients are completely decoupled with decreasing the temperature, suggesting the violation of the SE relation in supercooled water. Furthermore, the SED relations $\tau_{\ell} T / \eta$ and $D_{\mathrm{t}} \tau_{\ell}$ were investigated. We determined that these SED relations exhibit a strong dependence on the order $\ell$ of the Legendre polynomial, i.e., the examined angle of molecular reorientations.

These assessments of the SE and SED relations enabled the classification of various characteristic timescales into just two classes; dominated by "mobile" and "immobile" molecules of dynamical heterogeneities, depending on the degree of coupling with the translational diffusion constant $D_{\mathrm{t}}$. Moreover, $D_{\mathrm{t}}$ is coupled with the H-bond lifetime $\tau_{\mathrm{HB}}$ that is governed by mobile molecules that exhibit large molecular displacement amplitudes. These coupling dynamics indicate the preservation of the SE relation, which was rationalized by the peak time of NGP, $\tau_{\text {ngp }}$, which characterize the mobile 
molecules of dynamic heterogeneities. In addition, the stress relaxation time $\tau_{\eta}$ and the rotational relaxation time $\tau_{1}$ that is accompanied by a large angle amplitude, show the temperature dependence similar to that of $D_{\mathrm{t}}$, which is also governed by mobile molecules.

In contrast, $\eta / T$ is proportional to the structural $\alpha$-relaxation time $\tau_{\alpha}$ that is quantified by the density correlation function. This implies the violation of the SE relation (the decoupling between $\eta / T$ and $D_{\mathrm{t}}$ ) that are related to immobile molecules of dynamic heterogeneities. In fact, the peak time of NNGP, $\tau_{\text {nngp }}$ that characterize the contribution of immobile molecules shows the temperature dependence similar to that of the violation of the SE relation. As discussed, the shear viscosity $\eta$ is represented by $\eta \approx G_{\mathrm{p}} \tau_{\eta} \Gamma\left(1 / \beta_{\eta}\right) / \beta_{\eta}$ using the Green-Kubo formula [51]. The violation of the SE relation is thus expressed by $D_{\mathrm{t}} \eta / T \propto G_{\mathrm{p}} \Gamma\left(1 / \beta_{\eta}\right) /\left(T \beta_{\eta}\right)$, using the observed relationships, $D_{\mathrm{t}}^{-1} \propto \tau_{\mathrm{HB}}$ and $D_{\mathrm{t}}^{-1} \propto \tau_{\eta}$. Furthermore, we show that the rotational relaxation time for a small-angle amplitude, $\tau_{6}$, and the peak times of both translational and the peak times of rotational four-point correlation functions, $\tau_{\mathrm{t}}$ and $\tau_{\mathrm{r}}$, are also governed by immobile molecules. These are decoupled with $D_{\mathrm{t}}$, but instead exhibit the temperature dependence similar to $\eta / T$.

The identification of timescales that appropriately characterize the transport coefficients, $D_{\mathrm{t}}$ and $\eta$, paves the way to a deeper understanding of the violation of SE relation that is generally observed in various glass-forming liquids. In particular, the violation of the SE relation is directly relevance to the decoupling between the $\alpha$ relaxation time $\tau_{\alpha}$ and both the stress relaxation time $\tau_{\eta}$ and the H-bond lifetime $\tau_{\mathrm{HB}}$ in supercooled water. Further investigations should be undertaken in order to clarify this issue and obtain a unified description of structural relaxation in glass-forming liquids.

\section{Acknowledgments}

We thank K. Miyazaki, N. Matubayasi, T. Nakamura, and H. Shiba for valuable discussions. This work was partly supported by JSPS KAKENHI Grant Numbers JP15H06263, JP16H06018, and JP18H01188. The numerical calculations were performed at the Research Center of Computational Science, Okazaki, Japan.

\section{References}

[1] Ediger M D, Angell C A and Nagel S R Supercooled Liquids and Glasses 1996 J. Phys. Chem. 100, 13200-13212

[2] Ediger M D Spatially heterogeneous dynamics in supercooled liquids. 2000 Annu. Rev. Phys. Chem. $\mathbf{5 1}, 99-128$

[3] Angell C Perspective on the glass transition 1988 J. Phys. Chemi. Solids 49, 863-871

[4] Debenedetti P G and Stillinger F H Supercooled liquids and the glass transition 2001 Nature 410, 259-267

[5] Fujara F, Geil B, Sillescu H and Fleischer G Translational and rotational diffusion in supercooled orthoterphenyl close to the glass transition 1992 Z. Phys. B 88, 195 
[6] Cicerone M T and Ediger M D Enhanced translation of probe molecules in supercooled o-terphenyl: Signature of spatially heterogeneous dynamics? 1996 J. Chem. Phys. 104, 7210

[7] Hodgdon J and Stillinger F Stokes-Einstein violation in glass-forming liquids 1993 Phys. Rev. E 48, 207-213

[8] Stillinger F and Hodgdon J Translation-rotation paradox for diffusion in fragile glass-forming liquids 1994 Phys. Rev. E 50, 2064-2068

[9] Tarjus G and Kivelson D Breakdown of the Stokes-Einstein relation in supercooled liquids $1995 \mathrm{~J}$. Chem. Phys. 103, 3071-3073

[10] Ngai K L Breakdown of Debye-Stokes-Einstein and Stokes-Einstein relations in glass-forming liquids: An explanation from the coupling model 2009 Philos. Mag. B 79, 1783-1797

[11] Hansen J P and McDonald I R 2006 Theory of Simple Liquids, Third Edition (London: Academic Press)

[12] Balucani U and Zoppi M 1995 Dynamics of the Liquid State Oxford series on neutron scattering in condensed matter, 10 (USA: Oxford University Press)

[13] Thirumalai D and Mountain R D Activated dynamics, loss of ergodicity, and transport in supercooled liquids 1993 Phys. Rev. E 47, 479-489

[14] Yamamoto R and Onuki A Dynamics of highly supercooled liquids: Heterogeneity, rheology, and diffusion 1998 Phys. Rev. E 58, 3515-3529

[15] Yamamoto R and Onuki A Heterogeneous diffusion in highly supercooled liquids 1998 Phys. Rev. Lett. 81, 4915-4918

[16] Horbach J and Kob W Static and dynamic properties of a viscous silica melt 1999 Phys. Rev. B 60, 3169-3181

[17] Bordat P, Affouard F, Descamps M and Müller-Plathe F The breakdown of the Stokes Einstein relation in supercooled binary liquids 2003 J. Phys.: Condens. Matter 15, 5397-5407

[18] Berthier L Time and length scales in supercooled liquids 2004 Phys. Rev. E 69, 020201(R)

[19] Kumar S K, Szamel G and Douglas J F Nature of the breakdown in the Stokes-Einstein relationship in a hard sphere fluid 2006 J. Chem. Phys. 124, 214501

[20] Kim K and Saito S Role of the Lifetime of Dynamical Heterogeneity in the Frequency-Dependent Stokes-Einstein Relation of Supercooled Liquids 2010 J. Phys. Soc. Jpn. 79, 093601

[21] Ikeda A and Miyazaki K Glass transition of the monodisperse Gaussian core model. 2011 Phys. Rev. Lett. 106, 015701

[22] Shi Z, Debenedetti P G and Stillinger F H Relaxation processes in liquids: Variations on a theme by Stokes and Einstein 2013 J. Chem. Phys. 138, 12A526

[23] Sengupta S, Karmakar S, Dasgupta C and Sastry S Breakdown of the Stokes-Einstein relation in two, three, and four dimensions $2013 \mathrm{~J}$. Chem. Phys. 138, 12A548

[24] Kawasaki T and Onuki A Slow relaxations and stringlike jump motions in fragile glass-forming liquids: Breakdown of the Stokes-Einstein relation 2013 Phys. Rev. E 87, 012312

[25] Kawasaki T, Kim K and Onuki A Dynamics in a tetrahedral network glassformer: Vibrations, network rearrangements, and diffusion $2014 \mathrm{~J}$. Chem. Phys. 140, 184502

[26] Henritzi P, Bormuth A, Klameth F and Vogel M A molecular dynamics simulations study on the relations between dynamical heterogeneity, structural relaxation, and self-diffusion in viscous liquids. 2015 J. Chem. Phys. 143, 164502

[27] Saw S and Harrowell P The geometric mean squared displacement and the Stokes-Einstein scaling in a supercooled liquid $2015 \mathrm{~J}$. Chem. Phys. 143, 244502

[28] Ozawa M, Kim K and Miyazaki K Tuning pairwise potential can control the fragility of glassforming liquids: from a tetrahedral network to isotropic soft sphere models $2016 \mathrm{~J}$. Stat. Mech. 2016, 074002

[29] Schober H R and Peng H L Heterogeneous diffusion, viscosity, and the Stokes-Einstein relation in binary liquids 2016 Phys. Rev. E 93, 052607

[30] Parmar A D S, Sengupta S and Sastry S Length-Scale Dependence of the Stokes-Einstein and Adam-Gibbs Relations in Model Glass Formers 2017 Phys. Rev. Lett. 119, 056001 
[31] Puosi F, Pasturel A, Jakse N and Leporini D Communication: Fast dynamics perspective on the breakdown of the Stokes-Einstein law in fragile glassformers 2018 J. Chem. Phys. 148, 131102

[32] Kämmerer S, Kob W and Schilling R Dynamics of the rotational degrees of freedom in a supercooled liquid of diatomic molecules 1997 Phys. Rev. E 56, 5450-5461

[33] Lombardo T G, Debenedetti P G and Stillinger F H Computational probes of molecular motion in the Lewis-Wahnstrom model for ortho-terphenyl. 2006 J. Chem. Phys. 125, 174507

[34] Chong S H and Kob W Coupling and Decoupling between Translational and Rotational Dynamics in a Supercooled Molecular Liquid 2009 Phys. Rev. Lett. 102, 392

[35] Chen S H, Mallamace F, Mou C Y, Broccio M, Corsaro C, Faraone A and Liu L The violation of the Stokes-Einstein relation in supercooled water. 2006 Proc. Natl. Acad. Sci. U.S.A. 103, 12974-12978

[36] Becker S R, Poole P H and Starr F W Fractional Stokes-Einstein and Debye-Stokes-Einstein relations in a network-forming liquid. 2006 Phys. Rev. Lett. 97, 055901

[37] Kumar P Breakdown of the Stokes-Einstein relation in supercooled water 2006 Proc. Natl. Acad. Sci. U.S.A. 103, 12955-12956

[38] Kumar P, Buldyrev S V, Becker S R, Poole P H, Starr F W and Stanley H E Relation between the Widom line and the breakdown of the Stokes-Einstein relation in supercooled water 2007 Proc. Natl. Acad. Sci. U.S.A. 104, 9575-9579

[39] Mazza M G, Giovambattista N, Stanley H E and Starr F W Connection of translational and rotational dynamical heterogeneities with the breakdown of the Stokes-Einstein and StokesEinstein-Debye relations in water. 2007 Phys. Rev. E 76, 031203

[40] Xu L, Mallamace F, Yan Z, Starr F W, Buldyrev S V and Eugene Stanley H Appearance of a fractional Stokes-Einstein relation in water and a structural interpretation of its onset 2009 Nat. Phys. 5, 565-569

[41] Banerjee D, Bhat S N, Bhat S V and Leporini D ESR evidence for 2 coexisting liquid phases in deeply supercooled bulk water 2009 Proc. Natl. Acad. Sci. U.S.A. 106, 11448-11453

[42] Mallamace F, Branca C, Corsaro C, Leone N, Spooren J, Stanley H E and Chen S H Dynamical Crossover and Breakdown of the Stokes-Einstein Relation in Confined Water and in MethanolDiluted Bulk Water 2010 J. Phys. Chem. B 114, 1870-1878

[43] Jana B, Singh R S and Bagchi B String-like propagation of the 5-coordinated defect state in supercooled water: molecular origin of dynamic and thermodynamic anomalies 2011 Phys. Chem. Chem. Phys. 13, 16220-16226

[44] Qvist J, Mattea C, Sunde E P and Halle B Rotational dynamics in supercooled water from nuclear spin relaxation and molecular simulations 2012 J. Chem. Phys. 136, 204505

[45] Rozmanov D and Kusalik P G Transport coefficients of the TIP4P-2005 water model $2012 \mathrm{~J}$. Chem. Phys. 136, 044507

[46] Bove L E, Klotz S, Strässle T, Koza M, Teixeira J and Saitta A M Translational and Rotational Diffusion in Water in the Gigapascal Range 2013 Phys. Rev. Lett. 111, 185901

[47] Dehaoui A, Issenmann B and Caupin F Viscosity of deeply supercooled water and its coupling to molecular diffusion. 2015 Proc. Natl. Acad. Sci. U.S.A. 112, 12020-12025

[48] Guillaud E, Merabia S, de Ligny D and Joly L Decoupling of viscosity and relaxation processes in supercooled water: a molecular dynamics study with the TIP4P/2005f model 2017 Phys. Chem. Chem. Phys. 19, 2124-2130

[49] Guillaud E, Joly L, de Ligny D and Merabia S Assessment of elastic models in supercooled water: A molecular dynamics study with the TIP4P/2005f force field 2017 J. Chem. Phys. 147, 014504

[50] Galamba N On the hydrogen-bond network and the non-Arrhenius transport properties of water. 2017 J. Phys.: Condens. Matter 29, 015101

[51] Kawasaki T and Kim K Identifying time scales for violation/preservation of Stokes-Einstein relation in supercooled water 2017 Sci. Adv. 3, e1700399

[52] Shi R, Russo J and Tanaka H Origin of the emergent fragile-to-strong transition in supercooled water 2018 Proc. Natl. Acad. Sci. U.S.A. 115, 9444-9449 
[53] Montero de Hijes P, Sanz E, Joly L, Valeriani C and Caupin F Viscosity and self-diffusion of supercooled and stretched water from molecular dynamics simulations 2018 J. Chem. Phys. 149, 094503

[54] Saito S, Bagchi B and Ohmine I Crucial role of fragmented and isolated defects in persistent relaxation of deeply supercooled water 2018 J. Chem. Phys. 149, 124504

[55] Kawasaki T and Kim K Resolving spurious violation of the Stokes-Einstein-Debye relation in supercooled water 2018 arXiv (Preprint 1811.00373v2)

[56] Yamamoto R and Onuki A Kinetic Heterogeneities in a Highly Supercooled Liquid 1997 J. Phys. Soc. Jpn. 66, 2545-2548

[57] Shiba H, Kawasaki T and Onuki A Relationship between bond-breakage correlations and four-point correlations in heterogeneous glassy dynamics: configuration changes and vibration modes. 2012 Phys. Rev. E 86, 041504

[58] Shiba H, Yamada Y, Kawasaki T and Kim K Unveiling Dimensionality Dependence of Glassy Dynamics: 2D Infinite Fluctuation Eclipses Inherent Structural Relaxation 2016 Phys. Rev. Lett. 117, 245701

[59] Shiba H, Keim P and Kawasaki T Isolating long-wavelength fluctuation from structural relaxation in two-dimensional glass: cage-relative displacement 2018 J. Phys.: Condens. Matter 30, 094004

[60] Plimpton S Fast parallel algorithms for short-range molecular dynamics 1995 J. Comput. Phys. 117, $1-19$

[61] Abascal J L F and Vega C A general purpose model for the condensed phases of water: TIP4P/2005 2005 J. Chem. Phys. 123, 234505

[62] Abascal J L F and Vega C Widom line and the liquid-liquid critical point for the TIP4P/2005 water model 2010 J. Chem. Phys. 133, 234502

[63] Sumi T and Sekino H Effects of hydrophobic hydration on polymer chains immersed in supercooled water 2013 RSC Adv. 3, 12743-12750

[64] Overduin S D and Patey G N An analysis of fluctuations in supercooled TIP4P/2005 water 2013 J. Chem. Phys. 138, 184502

[65] De Marzio M, Camisasca G, Rovere M and Gallo P Mode coupling theory and fragile to strong transition in supercooled TIP4P/2005 water $2016 \mathrm{~J}$. Chem. Phys. 144, 074503

[66] Hamm P Markov state model of the two-state behaviour of water 2016 J. Chem. Phys. 145, 134501

[67] Singh R S, Biddle J W, Debenedetti P G and Anisimov M A Two-state thermodynamics and the possibility of a liquid-liquid phase transition in supercooled TIP4P/2005 water $2016 \mathrm{~J}$. Chem. Phys. 144, 144504

[68] Gonzalez M A, Valeriani C, Caupin F and Abascal J L F A comprehensive scenario of the thermodynamic anomalies of water using the TIP4P/2005 model 2016 J. Chem. Phys. 145, 054505

[69] Handle P H and Sciortino F Potential energy landscape of TIP4P/2005 water 2018 J. Chem. Phys. 148, 134505

[70] Rapaport D C Hydrogen bonds in water: Network organization and lifetimes 1983 Mol. Phys. 50, $1151-1162$

[71] Saito S and Ohmine I Translational and orientational dynamics of a water cluster (H2O)108 and liquid water: Analysis of neutron scattering and depolarized light scattering 1995 J. Chem. Phys. 102, 3566

[72] Luzar A and Chandler D Hydrogen-bond kinetics in liquid water 1996 Nature 379, 55-57

[73] Luzar A and Chandler D Effect of Environment on Hydrogen Bond Dynamics in Liquid Water 1996 Phys. Rev. Lett. 76, 928-931

[74] Luzar A Resolving the hydrogen bond dynamics conundrum 2000 J. Chem. Phys. 113, 10663

[75] Kumar R, Schmidt J R and Skinner J L Hydrogen bonding definitions and dynamics in liquid water 2007 J. Chem. Phys. 126, 204107

[76] Toninelli C, Wyart M, Berthier L, Biroli G and Bouchaud J P Dynamical susceptibility of glass formers: Contrasting the predictions of theoretical scenarios 2005 Phys. Rev. E 71, 041505 
[77] Rahman A Correlations in the Motion of Atoms in Liquid Argon 1964 Phys. Rev. 136, A405-A411

[78] Kob W, Donati C, Plimpton S J, Poole P H and Glotzer S C Dynamical Heterogeneities in a Supercooled Lennard-Jones Liquid 1997 Phys. Rev. Lett. 79, 2827-2830

[79] Flenner E and Szamel G Relaxation in a glassy binary mixture: Mode-coupling-like power laws, dynamic heterogeneity, and a new non-Gaussian parameter 2005 Phys. Rev. E 72, 011205 